\title{
The need for cataract surgery: projections based on lens opacity, visual acuity, and personal concern
}

\author{
Cathy A McCarty, Jill E Keeffe, Hugh R Taylor
}

\begin{abstract}
Aim-To assess the projected needs for cataract surgery by lens opacity, visual acuity, and patient concern.

Methods-Data were collected as part of the Melbourne Visual Impairment Project, a population based study of age related eye disease in a representative sample of Melbourne residents aged 40 and over. Participants were recruited by a household census and invited to attend a local screening centre. At the study sites, the following data were collected: presenting and best corrected visual acuity, visual fields, intraocular pressure, satisfaction with current vision, personal health history and habits, and a standardised eye examination and photography of the lens and fundus. Lens photographs were graded twice and adjudicated to document lens opacities. Cataract was defined as nuclear greater than or equal to standard $2,4 / 16$ or greater cortical opacity, or any posterior subcapsular opacities.
\end{abstract}

Results-3271 (83\% response) people living in their own homes were examined. The participants ranged in age from 40 to 98 years and $1511(46.2 \%)$ were men. Previous cataract surgery had been performed in $107(3.4 \%)$ of the participants. The overall prevalence of any type of cataract that had not been surgically corrected was $18 \%$. If the presence of cataract as defined was considered the sole criterion for cataract surgery with no reference to visual acuity, there would be 309 cataract operations per 1000 people aged 40 and over (96 eyes of people who were not satisfied with their vision, 210 eyes of people who were satisfied with their vision, and three previous cataract operations). At a visual acuity criterion of less than 6/12 (the vision required to legally drive a car), 48 cataract operations per 1000 would occur and people would be twice as likely to report dissatisfaction with their vision. Conclusions-Estimates of the need for cataract surgery vary dramatically by level of lens opacity, visual acuity, and patient concern. These data should be useful for the planning of health services. (Br f Ophthalmol 1999;83:62-65)

We have shown previously that the volume of cataract surgery in Australia increased 2.6 times in the years 1985 to $1994 .{ }^{1}$ The exact cost of cataract surgery in Australia is unknown but estimates for the USA for the year 1991 were $\$ 3.4$ billion for typical cases ${ }^{2}$ and costs are likely to rise in the USA and in other developed countries to meet increases in the demand for cataract surgery concomitant with the relative aging of the populations. A decrease in the threshold visual acuity for cataract surgery has been shown in the UK to double the number of people potentially eligible for cataract surgery, ${ }^{34}$ thus doubling the costs. The Preferred Practice Pattern of the Royal Australian College of Ophthalmologists for cataract surgery states that the "indications for surgery are dependent on various levels of visual impairment and cataract surgery is justified and appropriate when the subjective, objective, and education criteria are met", but does not have a visual acuity threshold at which cataract surgery is not advised. ${ }^{5}$ There has also been much discussion about the relative merit and cost of second eye cataract surgery. ${ }^{67}$

The influence of visual acuity, visual function, and surgeon on the demand for cataract surgery has been studied in a number of countries, with the general conclusion that functional impact and concern, not surgeon preference or visual acuity, are the major determinants of demand. ${ }^{8-11}$ The purpose of this study was to predict the number of cataract operations per 1000 people at risk that may be needed in Australia (and similar countries) by level of visual acuity and patient concern. These data will be useful for healthcare administrators to estimate demand for cataract services.

\section{Methods}

STUDY POPULATION

Details of the Melbourne Visual Impairment Project methodology have been published previously. ${ }^{12}$ In brief, nine pairs of census collector districts from the Melbourne Statistical Division were randomly selected from which to recruit residents who had resided in their homes for at least 6 months to attend a local screening centre.

STUDY PROTOCOL

Procedures at the local screening centre included assessment of presenting and best corrected distance visual acuity, ${ }^{13}$ reading vision, visual fields, intraocular pressure, fundus and lens photography, a standardised clinical slit lamp examination, a detailed interview about medical history and personal health habits. People were asked to rate their personal satisfaction with their current vision (with
Accepted for publication 29 July 1998 
glasses if used) as very dissatisfied, dissatisfied, satisfied or very satisfied. These responses were then classified in to the two categories of "dissatisfied" or "satisfied" for the analyses. All participants gave written informed consent and the study protocol was approved by the Royal Victorian Eye and Ear Hospital human research ethics committee.

Lens opacities were graded clinically at the time of the examination and subsequently from photos using the Wilmer cataract photo grading system. ${ }^{14}$ Cortical opacities were assessed with retroillumination and measured as the proportion in 16ths of pupil circumference occupied by opacity. For these analyses, cortical cataract is defined as $4 / 16$ or greater opacity. The area of posterior subcapsular (PSC) opacities was measured in square millimetres. The presence of any PSC opacity was classified as cataract. Nuclear opacities were compared against four standard photographs and decimalised. For these analyses, nuclear cataract was defined as opacity equal to or greater than standard photo 2 . These definitions of cataract are similar to what has been used in previous population based studies.

A Topcon SL5 photo slit lamp with a 0.1 $\mathrm{mm}$ slit beam of $9.0 \mathrm{~mm}$ in height set to an incident angle of $30^{\circ}$ and Ektachrome 200 ASA colour slide film were used to photograph nuclear opacities. Cortical and PSC opacities were photographed with an Oxford retroillumination camera and T-Max 400 film.

All of the photos were graded independently by two trained research assistants and discrepancies were adjudicated by the ophthalmic research fellow. With the exception of ungradable or missing photos, the photo grades were used for analyses.

\section{DATA MANAGEMENT}

Interview data were entered directly into the computer. All other data were entered twice and verified. Analyses were performed in SAS Version 6.10 (SAS Institute Inc, Cary, NC, USA) and are documented in the SAS procedure manuals. A $\mathrm{p}$ value $<0.05$ was considered to be statistically significant. $\chi^{2}$ analyses were employed to evaluate significant univariate relation among categorical variables while backwards logistic regression was used to assess the independent effect of categorical variables in multivariate models.

\section{Results}

STUDY POPULATION

A total of 3271 eligible (83\%) residents participated. The only significant difference between attenders and non-attenders was language spoken at home; non-attenders were more likely to be non-English speakers. ${ }^{15}$ The following variables were used to compare participants and non-participants and participants with the census data from the Australian Bureau of Statistics: age, sex, education, ethnicity. The study cohort has been shown to be representative of all Melburnians aged 40 and up. ${ }^{15}$ The participants ranged in age from 40 to 98 years (mean 58.7, SD 11.4) and 1511 $(46.2 \%)$ were men. Photos were used to classify cortical and PSC opacities in 2919 (90\%) right eyes and 2891 (89\%) left eyes. Photos were used to classify nuclear cataract in 2969 (92\%) right eyes and 2957 (91\%) left eyes. All available data are employed for each separate analysis.

\section{CATARACT PREVALENCE}

The overall prevalence of any type of cataract that had not been surgically removed was $18 \%$. The age and sex specific cataract status of each eye reveals the dramatic increase in cataract and cataract surgery with each decade of life (Table 1). Previous cataract surgery had been performed in 107 (3.4\%) of the participants; of the 103 with available information on their cataract surgery, $18 \%$ had occurred in the previous year. Controlling for age, women were significantly more likely to have cataract than men $(\mathrm{OR}=1.45,95 \% \mathrm{CL}=1.18,1.78)$.

RELATION OF LENS OPACITIES TO VISUAL ACUITY AND PERSONAL SATISFACTION WITH VISION

People who were dissatisfied with their vision were more likely to have cataract $\left(\chi^{2}, 1 \mathrm{df}\right.$ $=22.0, \mathrm{p}=0.001)$ and this was true at nearly all levels of best corrected visual acuity. It is interesting to note that 462 of 2300 (11.9\%) people with $6 / 6$ or better visual acuity reported dissatisfaction with their vision and that for the majority of these people, their dissatisfaction would not be attributed to cataract.

A backwards multivariate logistic regression model was fitted to predict dissatisfaction with current vision. The following variables were included as potential predictors: 10 year age group, lines of best corrected visual acuity (Snellen equivalent from $\log$ MAR), presence

Table 1 Prevalence of cataract ${ }^{\star}$ and previous cataract surgery by age and sex

\begin{tabular}{|c|c|c|c|c|c|c|c|}
\hline Age & Sex & $\begin{array}{l}\text { No cataract in } \\
\text { either eye }\end{array}$ & $\begin{array}{l}\text { Prevalent cataract in } \\
\text { one eye only }\end{array}$ & $\begin{array}{l}\text { Prevalent cataract in } \\
\text { both eyes }\end{array}$ & $\begin{array}{l}\text { Prevalent cataract in } \\
\text { one eye, previous } \\
\text { surgery in } 2 \text { nd eye }\end{array}$ & $\begin{array}{l}\text { Previous surgery in one } \\
\text { eye, no cataract in } 2 \text { nd } \\
\text { eye }\end{array}$ & $\begin{array}{l}\text { Previous surgery in } \\
\text { both eyes }\end{array}$ \\
\hline \multirow[t]{2}{*}{$40-49$} & Men, $n=352$ & $339(96.3 \%)$ & $8(2.27 \%)$ & $1(0.26 \%)$ & $0(0 \%)$ & $3(0.85 \%)$ & $1(0.28 \%)$ \\
\hline & Women, $n=459$ & $447(97.4 \%)$ & $10(2.18 \%)$ & $2(0.44 \%)$ & $0(0 \%)$ & $0(0 \%)$ & $0(0 \%)$ \\
\hline \multirow[t]{2}{*}{$50-59$} & Men, $n=442$ & $406(91.9 \%)$ & $24(5.4 \%)$ & $9(2.04 \%)$ & $2(0.45 \%)$ & $0(0 \%)$ & $1(0.23 \%)$ \\
\hline & Women, $n=524$ & $489(93.3 \%)$ & $20(3.82 \%)$ & $12(2.29 \%)$ & $2(0.38 \%)$ & $0(0 \%)$ & $1(0.19 \%)$ \\
\hline \multirow[t]{2}{*}{$60-69$} & Men, $n=423$ & $331(78.3 \%)$ & $35(8.27 \%)$ & $49(11.6 \%)$ & $3(0.71 \%)$ & $1(0.24 \%)$ & $4(0.95 \%)$ \\
\hline & Women, $n=429$ & $304(70.9 \%)$ & $53(12.4 \%)$ & $61(14.2 \%)$ & $4(0.93 \%)$ & $2(0.47 \%)$ & $5(1.17 \%)$ \\
\hline \multirow[t]{2}{*}{$70-79$} & Men, $n=218$ & $117(53.7 \%)$ & $22(10.1 \%)$ & $61(28.0 \%)$ & $6(2.75 \%)$ & $2(0.92 \%)$ & $10(4.59 \%)$ \\
\hline & Women, $n=220$ & $87(40.0 \%)$ & $33(15.0 \%)$ & $86(39.1 \%)$ & $6(2.73 \%)$ & $2(0.91 \%)$ & $6(2.73 \%)$ \\
\hline \multirow[t]{2}{*}{$80-89$} & Men, $n=56$ & $12(21.4 \%)$ & $8(14.3 \%)$ & $22(39.3 \%)$ & $7(12.5 \%)$ & $1(1.79 \%)$ & $6(10.7 \%)$ \\
\hline & Women, $\mathrm{n}=86$ & $4(4.65 \%)$ & $7(8.14 \%)$ & $51(59.3 \%)$ & $10(11.6 \%)$ & $2(2.33 \%)$ & $12(14.0 \%)$ \\
\hline \multirow[t]{2}{*}{$90+$} & Men, $n=3$ & $0(0 \%)$ & $0(0 \%)$ & $3(100 \%)$ & $0(0 \%)$ & $0(0 \%)$ & $0(0 \%)$ \\
\hline & Women, $\mathrm{n}=12$ & $0(0 \%)$ & $1(8.33 \%)$ & $4(33.3 \%)$ & $2(16.7 \%)$ & $0(0 \%)$ & $5(41.7 \%)$ \\
\hline All ages & Total, $\mathrm{n}=3224$ & $2536(78.7 \%)$ & $221(6.85 \%)$ & $361(11.2 \%)$ & $42(1.3 \%)$ & $13(0.40 \%)$ & 51 \\
\hline
\end{tabular}

^Nuclear opacity $\geqslant$ photo standard $2, \geqslant 4 / 16$ cortical opacity, any PSC. 


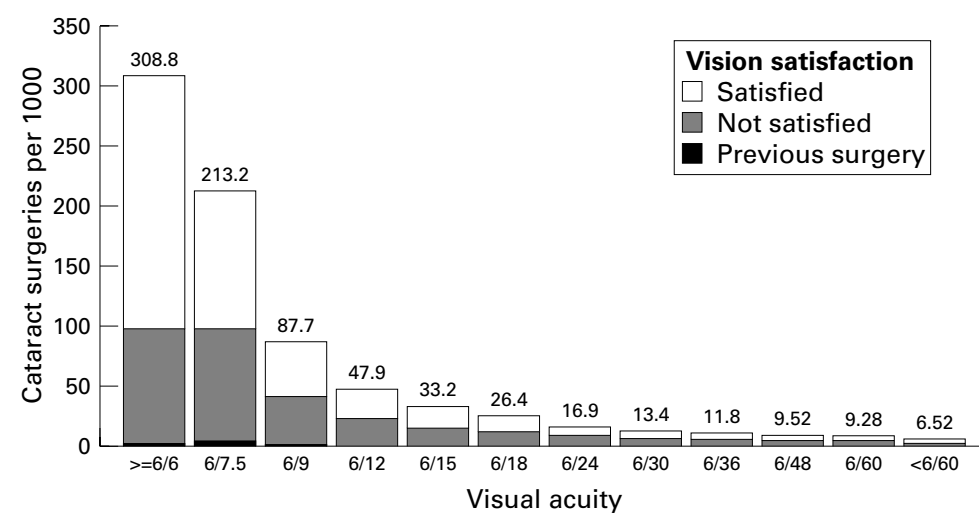

Figure 1 Cumulative number of cataract (defined as nuclear opacity $\geqslant$ photo standard 2, $\geqslant 4 / 16$ cortical opacity or any posterior subcapsular opacities (PSC) operations required per 1000 population aged 40+ by level of best corrected visual acuity and patient satisfaction with vision. of referral for cataract surgery by Australian ophthalmologists is patient concern. ${ }^{8}$

The Royal Australian College of Ophthalmologists acknowledge that although "no single eye test adequately describes the effect of cataracts on a patient's visual status or functional ability", "simple Snellen visual acuity is the most universally used index of visual function". However, as we and other researchers have shown, visual acuity is not highly related to satisfaction with vision. A study of patients' perception of their need for cataract surgery conducted in Norway revealed that visual function and visual acuity were not highly correlated until visual acuity was less than $6 / 24$ in the worse eye. ${ }^{16}$ The VF- $14,{ }^{17}$ an index of functional impairment in patients with cataract, has recently been used with cataract patients in the USA. Researchers have shown that the VF-14 is a better measure of improvement in visual function after cataract surgery than visual acuity. ${ }^{18}$

Another important reason to consider patient concern with vision simultaneously with visual acuity is the expected improvement in outcomes with cataract surgery. Several studies have now shown that visual function before surgery is the strongest predictor of visual outcome and quality of life after cataract surgery, independent of visual acuity. ${ }^{19-22}$ This implies that if cataract surgery were advised solely on the basis of visual acuity, the change in satisfaction and visual outcome would not be as great. Our data support this hypothesis; at better levels of visual acuity, the majority of people are satisfied with their vision and would not be expected to benefit substantially from cataract surgery.

As cataract surgery improves, giving better visual results with fewer adverse reactions, the procedure is done for less severe visual impairment. Our data are consistent with a doubling of the need for cataract surgery with each two line change in visual acuity. The change from aphakic ICCE to ECCE/IOL was associated with a drop in the visual acuity threshold from $6 / 60$ to $6 / 18$. The use of phacoemulsification is now further lowering that threshold to $6 / 6$, $6 / 7.5$ or $6 / 9$.

The level of lens opacity, visual acuity, and visual function all need to be considered in the recommendation of patients for cataract surgery. The establishment or enforcement of guidelines and thresholds for cataract surgery will have obvious implications for total costs to the community, the expected size of improvement in outcomes, the cost utility and cost effectiveness of the procedure, and the size of the waiting lists at public hospitals. The information from this study should assist in the planning of services to address the expected increase in demand for cataract surgery in developed countries.

The Melbourne Visual Impairment Project was funded in part by grants from the National Health and Medical Research Council, the Victorian Health Promotion Foundation, the Ansell Ophthalmology Foundation, and the Dorothy Edols Estate. Kodak Australia donated slide film for the project. umphrey Zeiss donated the autorefractor for use in the study. The authors acknowledge the contributions of the following individuals: Ms Sharon Bayley, Ms Marie Bissinella, Dr Charles

level of satisfaction with vision in conjunct with lens opacity and visual acuity as we have shown previously that the strongest predictor 
Guest, Ms Cara Jin, Dr Patricia Livingston, Ms Claire McKean, Dr Yury Stanislavsky, Ms Cathy Walker, and $\mathrm{Mr}$ Matthew Wensor.

1 Keeffe JE, Taylor HR. Cataract surgery in Australia 1985-94. Aust NZ f Ophthalmol 1996;24:313-17.

2 Steinberg EP, Javitt JC, Sharkey PD, et al. The content and cost of cataract surgery. Arch Ophthalmol 1993;111:1041cost

3 Desai P. The National Cataract Surgery Survey: III Process features. Eye 1993;7:667-71.

4 Wormald RPL, Evans JR. When does a lens opacity become a cataract? Estimating the effect of lowering the threshold for intervention on demand for services. Invest Ophthalmol Vis Sci 1993(Suppl);35:1963.

5 The Royal Australian College of Ophthalmologists. Preferred practice patterns. Cataract and intraocular lens surgery in the otherwise health adult eye. Sydney: Royal Australian College of Ophthalmologists, 1995.

6 Javitt JC, Brenner H, Curbow B, et al. Outcomes of cataract surgery. Improvements in visual acuity and subjective visual function after surgery in the first, second, and both eyes. Arch Ophthalmol 1993;111:686-91.

7 Javitt JC, Steinberg EP, Sharkey P, et al. Cataract surgery in Javitt JC, Steinberg EP, Sharkey P, et al. Cataract surgery in one eye or both.

8 Keeffe JE, McCarty CA, Chang WP, et al. Relative importance of VA, patient concern and patient lifestyle on referral for cataract surgery. Invest Ophthalmol Vis Sci 1996; 37:S183

9 Escarce JJ. Would eliminating differences in physician practice style reduce geographic variations in cataract surgery rates? Med Care 1993;12:1106-18

10 Curbow B, Legro MW, Brenner MH. The influence of patient-related variables in the timing of cataract extraction. Am $\mathcal{F}$ Ophthalmol 1993;115:614-22.

11 Mordue A, Parkin DW, Baxter C, et al. Thresholds for treatment in cataract surgery. F Publ Health Med 1994;16:393-
12 Livingston PM, Carson CA, Stanislavsky YL, et al. Methods for a population-based study of eye disease: the Melbourne Visual Impairment Project. Ophthalmic Epidemiol 1994;1: 139-48.

13 Taylor HR, Livingston PM, Stanislavsky YL, et al. Visual mpairment in Australia: distance visual acuity, near vision, and visual field findings of the Melbourne Visual Impairment Project. Am f Ophthalmol 1997;123:328-37.

14 Taylor HR, West SK. A simple system for the clinical grading of lens opacities. Lens Research 1988;5:175-81.

15 Livingston PM, Lee SE, McCarty CA, et al. A comparison of participants with non-participants in a population-based epidemiologic study: the Melbourne Visual Impairment Project. Ophthalmic Epidemiol 1997;4:73-81.

16 Sletteberg O, Høvding G, Bertelson T. Do we operate too many cataracts? The referred cataract patients' own appraisal of their need for surgery. Acta Ophthalmol Scand 1995;73:77-80.

17 Steinberg EP, Tielsch JM, Schein OD, et al. The VF-14. An index of functional impairment in patients with cataract. Arch Ophthalmol 1994;112:630-8.

18 Steinberg EP, Tielsch JM, Schein OD, J et al. National study of cataract surgery outcomes. Variation in 4-month postoperative oucomes as reflected in multiple outcome measures. Ophthalmology 1994;101:1131-41.

19 Elam JT, Graney MJ, Applegate WB, et al. Functional outcome one year following cataract surgery in elderly persons. come one year following cata

20 Brenner MH, Curbow B, Javitt JC, et al. Vision change and quality of life in the elderly. Response to cataract surgery and treatment of other chronic ocular conditions. Arch Ophthalmol 1993;111:680-5.

21 Mangione CM, Phillips RS, Lawrence MG, et al. Improved visual function and attenuation of declines in health-related quality of life after cataract extraction. Arch Ophthalmol 1994;112:1419-25.

22 Schein OD, Steinberg EP, Cassard SD, et al. Predictors of outcome in patients who underwent cataract surgery. $O p h$ thalmology 1995; 102:817-23. 